\title{
Subxiphoid single-incision thoracoscopic surgery for bilateral primary spontaneous pneumothorax
}

\author{
Chao-Yu Liư ${ }^{1,2}$, Chen-Sung Lin"2,3, Chia-Chuan Liư \\ ${ }^{1}$ Division of Thoracic Surgery, Department of Surgery, Far Eastern Memorial Hospital, New Taipei City, Taiwan \\ ${ }^{2}$ School of Medicine, National Yang-Ming University, Taipei, Taiwan \\ ${ }^{3}$ Division of Thoracic Surgery, Department of Surgery, Taipei Hospital, Ministry of Health and Welfare, New Taipei City, Taiwan \\ ${ }^{4}$ Department of Surgery, Koo-Foundation Sun Yat-Sen Cancer Center, Taipei, Taiwan
}

Videosurgery Miniinv 2015; 10 (1): 125-128

DOI: $10.5114 /$ wiitm.2015.48572

\begin{abstract}
It has been reported that single-incision thoracoscopic surgery can reduce postoperative pain without compromising the main surgical steps required for treating patients affected by primary spontaneous pneumothorax. However, all the reported thoracoscopic surgery cases with a single-incision procedure were via the intercostal route for unilateral pulmonary lesions. We present a novel single-incision thoracoscopic technique via a subxiphoid route to perform onestage bilateral thoracoscopic surgery for bilateral spontaneous pneumothorax. Reduced postoperative pain, shorter operative time, and better cosmetic results are potential benefits of this technique in selected patients. The subxiphoid single-incision procedure may be indicated in patients with bilateral pulmonary lesions requiring surgical resections.
\end{abstract}

Key words: pneumothorax, subxiphoid, single-incision.

\section{Introduction}

There has been a trend toward fewer surgical incisions in thoracic surgery. It has been widely accepted that minimally invasive surgery is adopted in daily practice for the thoracic surgeon nowadays. Single-incision video-assisted thoracoscopic surgery (VATS) has been proposed as a feasible approach in both diagnostic and therapeutic purposes in cancerous and non-cancerous diseases [1]. For primary spontaneous pneumothorax (PSP), it has been reported that the single-incision VATS can reduce postoperative pain without compromising any of the main surgical steps required for treating patients affected by PSP [2]. However, all the reported single-incision VATS were via the intercostal route for unilateral pulmonary lesions. We describe a novel single-incision VATS via a subxiphoid route for bilateral PSP.

\section{Case report}

A 21-year-old man with a known history of recurrent left side PSP (Photo $1 \mathrm{~A}$ ) was found to have a right side PSP (Photo $1 \mathrm{~B}$ ). Chest computed-tomography $(\mathrm{CT})$ revealed multiple and grouped subcentimeter blebs with variable size in the apical portion of the right lung (Photo $1 \mathrm{C}$, arrow). He had not received surgical intervention for his left side recurrent PSP and thus was admitted for surgery due to a contralateral recurrence of PSP. A one-staged bilateral surgical intervention was planned.

With the patient under general anesthesia and double-lumen endotracheal tube intubation, we positioned the patient supine with both arms $90^{\circ}$ abducted from the neutral position. A $3-\mathrm{cm}$ midline longitudinal incision was made immediately below the sternocostal triangle. The rectus abdominis was

\section{Address for correspondence}

Dr. Chia-Chuan Liu, Division of Thoracic Surgery, Department of Surgery, Koo-Foundation Sun Yat-Sen Cancer Center, 125 Lih-Der Road, Pei-Tou District, Taipei, Taiwan, phone: 88622897 0011, ext. 1760, fax: 88622858 6134, e-mail: gcliu@kfsyscc.org 

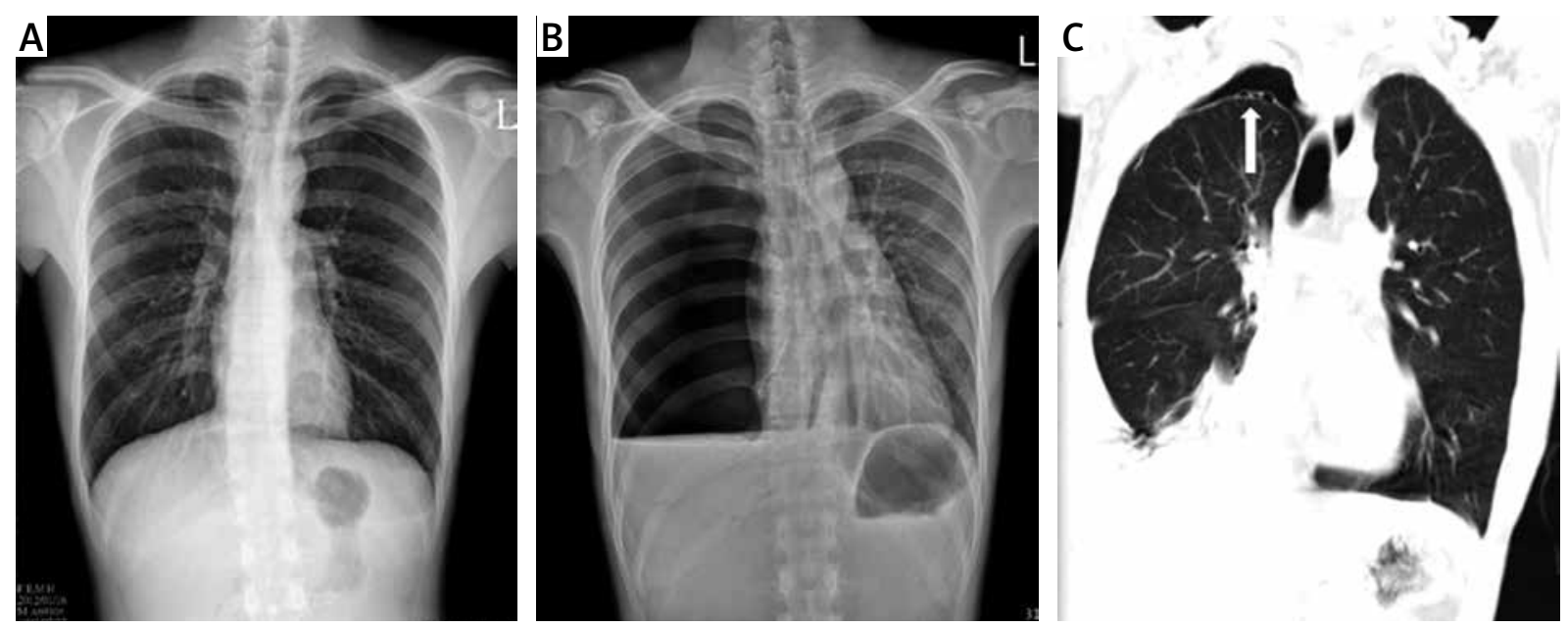

Photo 1. A - Patient has a history of left primary spontaneous pneumothorax (PSP) without previous surgical intervention. $\mathbf{B}$ - Contralateral recurrence of PSP associated with hemothorax. $\mathbf{C}-$ Chest CT revealed groups of blebs at apex of right lung (arrow)

divided at the midline to expose the xiphoid process. The rectus abdominis insertion to the xiphoid process was detached by an electric knife. After that, we could find the infrasternal angle which is formed by the xiphoid process and the subcostal margin (Photo $2 \mathrm{~A}$, arrow). The right side pleural cavity was opened by finger dissection via the infrasternal angle above the level of the diaphragm. A wound protector and a 5-mm 30-degree rigid endoscope (Karl-Storz Hopkins II Laparoscope) were used. Wedge resections of blebs by endocutters (Echelon Flex Powered Endopath Stapler) and abrasion pleurodesis were performed (Photo 3 A). An identical surgical procedure was performed to enter the left side chest cavity

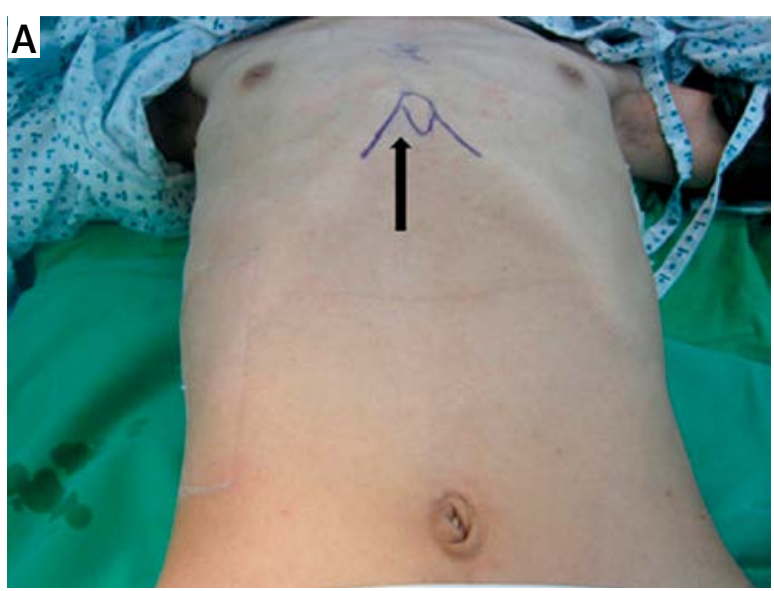

from the same subxiphoid incision (Photo $3 \mathrm{~B}$ ). After surgery, two $20 \mathrm{Fr}$ chest tubes were placed via the subxiphoid incision, with one to the right side and one to the left side chest cavity (Photo 2 B). The patient tolerated the whole procedure well, with only an occasional ventricular premature complex noted when performing the left side procedure. Chest tubes were removed on postoperative day 3 and the patient was discharged uneventfully.

\section{Discussion}

Single-incision VATS has been proposed as an alternative approach for thoracic diseases since 2004 [3].

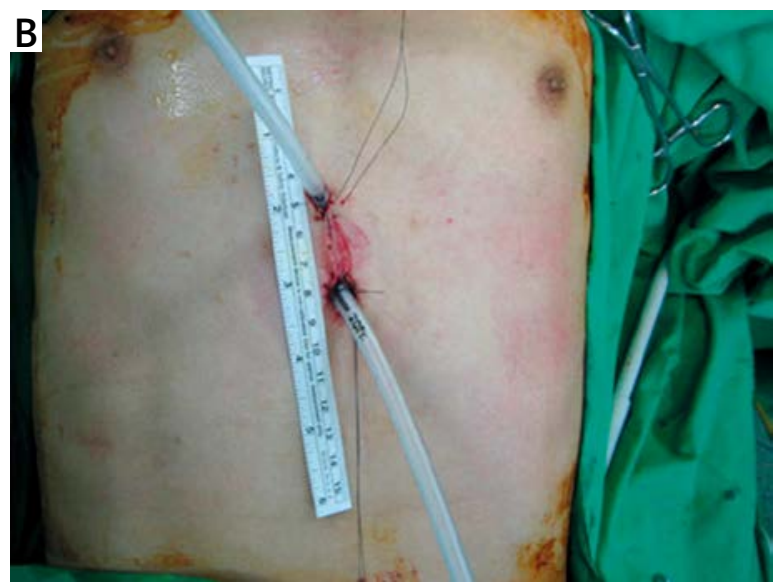

Photo 2. A - 3-cm longitudinal midline incision was made immediately below the sternocostal triangle (the level of the xiphoid process). The infrasternal angle (arrow) is the landmark for entry to bilateral chest cavities. B - Two chest tubes were placed via the incision with one to the right side (the lower one) and one to the left side (the upper one) chest cavity 

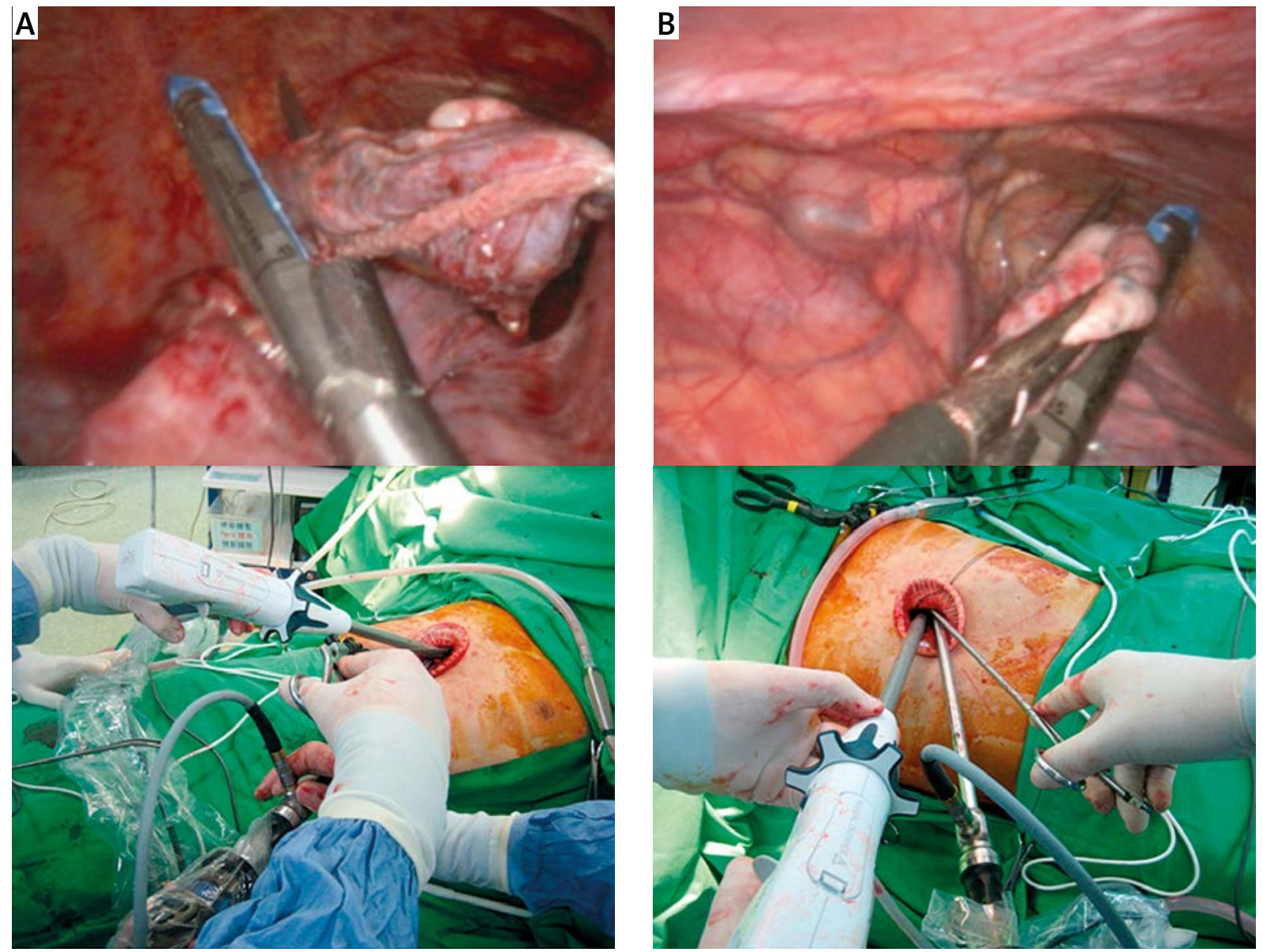

Photo 3. Instrumental positioning and intraoperative findings. A - For the right side procedure, operator and assistant stand on the left side of the patient with the table $30^{\circ}$ off center, tilted to the left. $\mathbf{B}-$ For the left side procedure, operator and assistant stand on the right side of the patient with the table tilted $30^{\circ}$ to the right

Although the history of single-incision VATS is less than a decade, more and more thoracic surgeons are interested in its benefit of reducing postoperative pain, better cosmesis, and a more natural angle of view for the surgical site [4]. For PSP, conventional two- or three-incision VATS has been performed widely as a standard procedure. However, Sihoe et al. reported that $52.9 \%$ of patients who had conventional multiple-port VATS for PSP complained of chest wall paresthesia which may be attributed to torquing of the camera or instruments in the ports during surgery [5]. To ameliorate the postoperative paresthesia, Rocco et al. proposed a uniportal VATS technique for PSP in 2005 and concluded that postoperative pain and paresthesia in uniportal VATS were lower than those in three-port VATS [3]. Salati et al., in 2008, also corroborated that uniportal VATS offered both lowered postoperative pain and reduced medical costs compared to the traditional three-port VATS technique for treating PSP [6]. Nevertheless, with the single-incision technique, the rate of postoperative pain and chest wall paresthesia might be lowered but still unavoidable. Furthermore, a contralateral recurrence may occur in up to $25 \%$ to $40 \%$ of patients with PSP [7]. A single-stage bilateral VATS may be indicated in selected patients to prevent contralateral recurrence [8]. In contrast to bilateral chest wall incisions, the subxiphoid single-incision technique, sparing one incision, may be more welcomed.

The potential benefits of the subxiphoid single-incision VATS technique for bilateral simultaneous PSP or PSP with contralateral recurrence may lie in two aspects: First, lowering postoperative pain and avoidance of chest wall paresthesia could be expected since there would be only one incision 
and the working space is away from the intercostal space. The injury to the intercostal nerve would be minimized. Second, the operation time would be shortened because there would be no need to reposition the patient when performing surgery on the contralateral side, and only a small utility incision needs to be closed at the end of surgery. However, this technique may have the following weaknesses: First, the subxiphoid route creates a caudal-cranial, anterior-posterior axis of view from the lower mediastinum of the chest cavity. Good traction would be needed to examine the lesions of the posterior aspect of the lung. Second, entering the left side chest cavity would compress the heart. Intraoperative arrhythmia would thus be encountered when inserting instruments into the left side chest cavity. Fortunately, most blebs or bulla in PSP were found in the apex of the upper lobe or superior segment of the lower lobe. These locations can be easily seen and approached via the subxiphoid technique. Additionally, patients with PSP are mostly slim and young, with a robust and normal size of the heart. The heart could be less compressed, and intraoperative arrhythmia, even if it occurred, might be better tolerated by these young and otherwise healthy patients.

To strengthen the weakness of the subxiphoid approach, operators would need to pass the learning curve required for the single-incision thoracoscopic technique. Good cooperation between an operator and assistants is a prerequisite to conquer the difficulties of single-incision VATS such as crowding and limited maneuverability of instruments. The use of specially designed long-curved with double-hinged instruments is a must for the development of this procedure. A thoracoscope which can provide a variable range of view could also help to examine the posterior aspect of the lung, especially posterior mediastinal lesions.

\section{Conclusions}

Overall, subxiphoid single-incision VATS may emerge as an alternative approach in patients with bilateral pulmonary lesions, especially indicated for treatment of simultaneous or contralateral recurrence PSP.

\section{Conflict of interest}

The authors declare no conflict of interest.

\section{References}

1. Rocco G, Martucci N, La Manna C, et al. Ten-year experience on 644 patients undergoing single-port (uniportal) video-assisted thoracoscopic surgery. Ann Thorac Surg 2013; 96: 434-38.

2. Jutley RS, Khalil MW, Rocco G. Uniportal vs standard three-port VATS technique for spontaneous pneumothorax: comparison of post-operative pain and residual paraesthesia. Eur J Cardiothorac Surg 2005; 28: 43-6.

3. Rocco G, Martin-Ucar A, Passera E. Uniportal VATS wedge pulmonary resections. Ann Thorac Surg 2004; 77: 726-8.

4. Calvin SH Ng. Uniportal VATS in Asia. J Thorac Dis 2013; 5 (Suppl. 3): S221-5.

5. Sihoe AD, Au SS, Cheung ML, et al. Incidence of chest wall paresthesia after video-assisted thoracic surgery for primary spontaneous pneumothorax. Eur J Cardiothorac Surg 2004; 25 : 1054-8.

6. Salati M, Brunelli A, Xiume F, et al. Uniportal video-assisted thoracic surgery for primary spontaneous pneumothorax: clinical and economic analysis in comparison to the traditional approach. Interact Cardiovasc Thorac Surg 2008; 7: 63-6.

7. de Hoyos A. Pneumothorax. In: General thoracic surgery. $7^{\text {th }}$ ed. Shields TW, LoCicero J, Reed CE, Feins RH (eds.). Lippincott Williams \& Wilkins, New York, NY 2009; 82833.

8. Huang TW, Lee SC, Cheng YL, et al. Contralateral recurrence of primary spontaneous pneumothorax. Chest 2007; 132: 1146-50.

Received: 3.10.2014, accepted: 14.12.2014. 